\title{
POWER LIMITATIONS IN BARITT DEVICES $\dagger$
}

\author{
S. P. KWOK $\ddagger$ and G. I. HADDAD \\ Electron Physics Laboratory, Department of Electrical and Computer Engineering, The University of Michigan, Ann \\ Arbor, MI 48109, U.S.A.
}

(Received 19 March 1975; in revised form 9 February 1976)

\begin{abstract}
The results of a large-signal numerical simulation of the BARITT device using a single-carrier model are given to illustrate the basic transport mechanisms involved in the device. The results of existing first-order small- and large-signal analyses are compared with those computed numerically. Space-charge-limited thermionic injection, spatial velocity modulation, carrier diffusion, premature carrier collection, large-signal electric field depression and the low-field drift region are found to account for the large reduction of power and efficiency of the BARITT device. The maximum power and efficiency of Si uniformly doped and Read structures are found to be approximately $300 \mathrm{~W} / \mathrm{cm}^{2}$ and $3 \%$, respectively. The effects of doping profile and material parameters are found to involve a trade off of large-signal handling capability and frequency of operation on one hand vs the negative conductance and thus the $Q$ factor of BARITT devices on the other hand. The excessively high electron mobility in $\mathrm{GaAs}$ is found to be detrimental to device operation.
\end{abstract}

\section{INTRODUCTION}

The objectives of this paper are to estimate the power capability and efficiency of BARITT devices and to compare the results of a large-signal computer simulation with the predictions of existing first-order theories in order to reveal the basic factors limiting the power and the efficiency of the device. The first-order large-signal calculations of power and efficiency reported by Rüegg[1] were chosen as a reference for comparison with numerical results obtained here. For convenience and emphasis the limiting assumptions embodied in this theory [1] are: (1) an in-phase impulse current injection of unlimited magnitude, (2) a constant saturated carrier drift velocity throughout the entire drift region and (3) the large-signal limitation due to avalanche breakdown at the maximum RF voltage and the minimum velocity-saturation field during the minimum $\mathrm{RF}$ voltage. That theory predicted that Si BARITT devices should be capable of generating a power output of $24,000 \mathrm{~W} / \mathrm{cm}^{2}$ with a maximum efficiency of $21 \%$ at $10 \mathrm{GHz}$ [1]. The experimentally reported values are, respectively, two orders and one order of magnitude smaller [2-4]. The computer simulation employed here is based on a single carrier model. Its numerical technique is similar to that reported by Scharfetter and Gummel [5]. The Einstein relation between mobility and diffusion coefficient is assumed throughout. To check the validity of the computer simulation, the numerical results of several test cases were found [4] to agree fairly well with the experimental findings reported by Snapp and Weissglass [2]. Despite its limitation, the small-signal analysis is particularly useful for the estimation of the frequency of operation and the maximum negative conductance of the BARITT device. Thus, for illustration the results of three simple analyses based on different assumptions are compared with those obtained numerically.

The paper commences with a brief description and a

tWork supported by the Air Force Office of Scientific Research, Air Force Systems Command, USAF, under Grant No. AFOSR-712035.

$\ddagger$ Presently at Hughes Research Laboratories, Torrance, CA 90505, U.S.A. comparison of small-signal conductances. It is followed by an approximate analysis which, under the large-signal condition, approaches asymptotically that given by Rüegg[1]. Then the results of computer simulation are given to illustrate the basic device mechanisms involved in the BARITT device. To estimate the power capability of BARITT devices the maximum power and efficiency of four $X$-band device structures: Si $p-n-p$ and its complementary $n-p-n, n-p-\nu-n$, and GaAs $n-p-n$ structures, are computed numerically. To reveal and quantify the basic factors that significantly degrade the power and efficiency of the BARITT device from the capabilities predicted by the first-order theory, the numerical results of the Si $n-p-\nu-n$ Read structure are used for comparison with those reported by Rüegg[1]. Finally, the effects of doping profile and material parameters upon the properties and in particular the power capability of BARITT devices are given.

\section{Small-signal analysis}

\section{FIRST-ORDER THEORY}

In order to generalize the small-signal theory given elsewhere [6-10] a $p^{+}-n_{1}-n_{2}-p^{+}$structure is considered. For a low-current injection level the electric field profile of the device arises mainly from the impurity ions as shown in Fig. 1 . The width of region 1 is assumed to be larger than its natural depletion width. The device is divided into two regions: the injection region $\left[0, x_{m}\right]$ and the drift region $\left[x_{m}\right.$, $w$ ]. Minority carriers are thermionically injected at the barrier maximum plane $\left(x=x_{m}\right)$ into the drift region where they drift with a constant velocity. By neglecting the impedance of the injection region it can be shown that the device impedance can be expressed as [9]

$$
\begin{aligned}
Z & =\frac{1}{\omega C}\left\{\frac{\sigma_{t h}}{\sigma_{t h}^{2}+(\omega \epsilon)^{2}}\left(\frac{\sigma_{t h}\left(1-\cos \theta_{t}\right)+\omega \epsilon \sin \theta_{t}}{\theta_{t}}\right)\right. \\
& \left.-i\left[1-\frac{\sigma_{t h}}{\sigma_{t h}^{2}+(\omega \epsilon)^{2}}\left(\frac{\sigma_{t h} \sin \theta_{t}-\omega \epsilon\left(1-\cos \theta_{t}\right)}{\theta_{t}}\right)\right]\right\},
\end{aligned}
$$




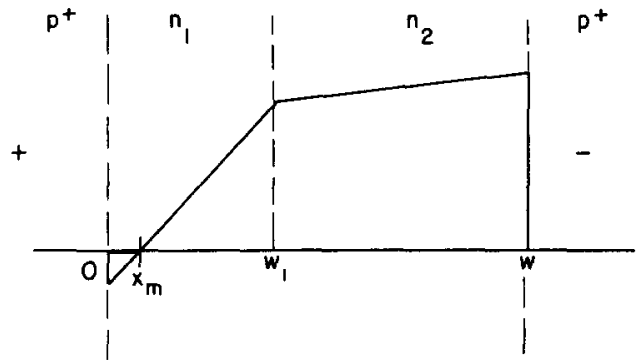

Fig. 1. Electric field profile of a $p^{+}-n_{1}-n_{2}-p^{+}$structure.

$$
\begin{aligned}
& \theta_{t}=\frac{\omega\left(w-x_{m}\right)}{\langle v\rangle}, \\
& C=\frac{\epsilon}{w-x_{m}}
\end{aligned}
$$

and $\langle v\rangle$ is the average drift velocity. The thermionic injection conductivity is given by the expression $[6,9]$

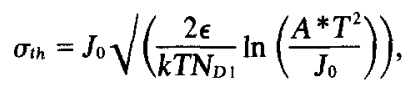

which depends on the doping density of the first region only [4]. As can be seen from eqn (1) the injection conductivity affects both the magnitude of the negative resistance and the frequency at which it occurs.

To illustrate the effects of nonsaturated drift velocity and the injection conductivity on the negative conductance of the BARITT device, several versions of first-order calculations made on a $\mathrm{Si} p^{+}-n-p^{+}$structure with a base impurity doping density of $3.3 \times 10^{15} / \mathrm{cm}^{3}$ and width equal to $6.0 \mu \mathrm{m}$ are given next.

The first version [6-8] divides the device into an injection region and a drift region. Carriers are injected thermionically at the barrier maximum plane into the drift region where they drift at the saturated velocity. This theory tends to predict a higher operating frequency of the device. To remedy this shortcoming the drift region is further subdivided into a low-field region, where mobility assumes its low-field constant value, and a high-field region, where the carrier velocity is saturated. The calculated negative conductance as a function of frequency at a bias current density of $50 \mathrm{~A} / \mathrm{cm}^{2}$ is plotted in Fig. 2, and the optimum negative conductance as a function of $\mathrm{dc}$ bias current density is given in Fig. 3. The introduction of the low-field drift region reduces somewhat the frequency of operation. However, the optimum negative conductance is not significantly affected.

A more dramatic change is obtained in the device impedance characteristics through the value of injection conductivity. Chu and Sze [9] in attempting to fit the theory with their experimental measurements introduced a spacecharge-limited conductance,

$$
\sigma_{s c}=\frac{2 \epsilon\langle v\rangle}{w},
$$

in parallel with the thermionic injection conductivity, $\sigma_{t h}$, resulting in an injection conductivity

$$
\sigma=\frac{\sigma_{t h} \sigma_{s c}}{\sigma_{t h}+\sigma_{s c}}
$$

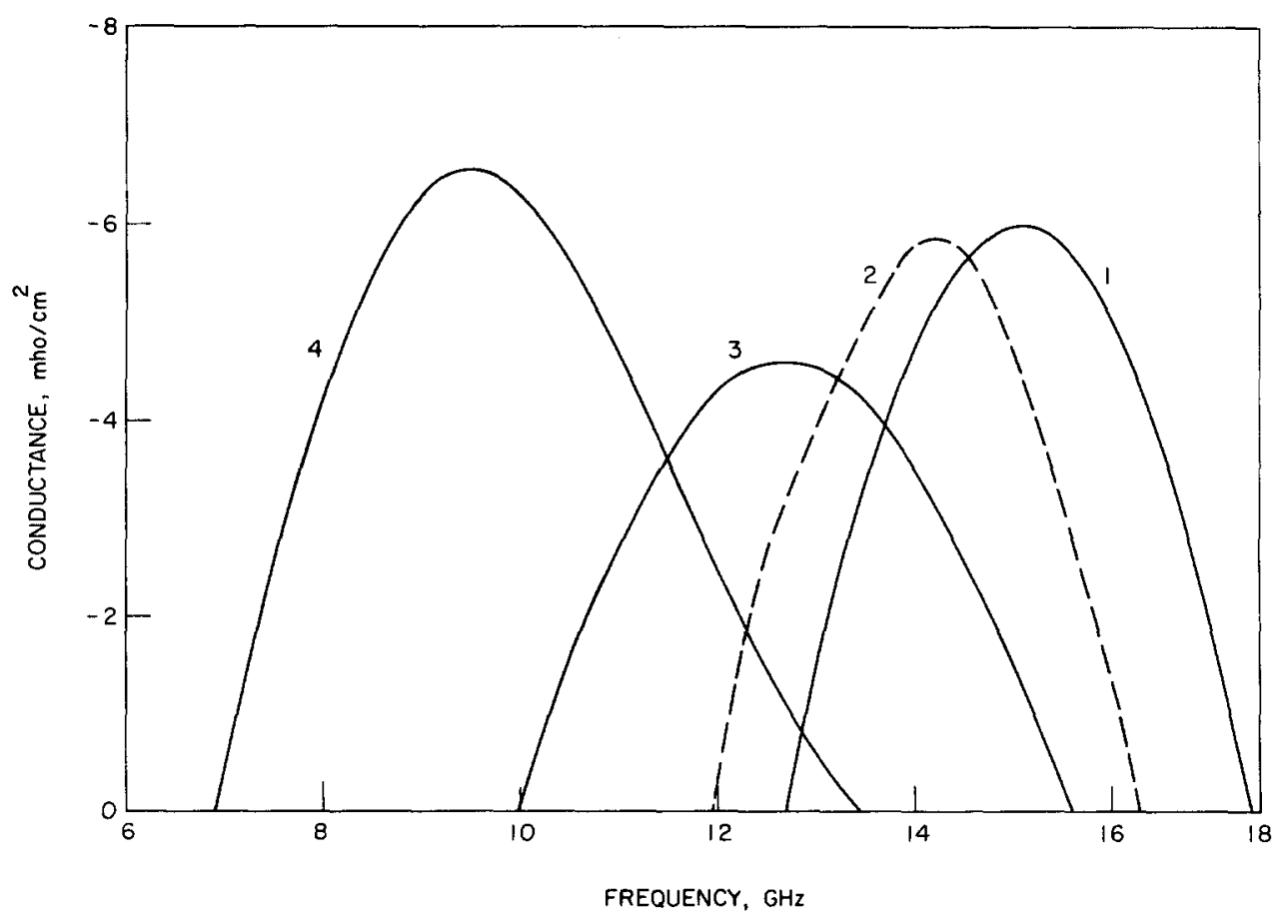

Fig. 2. Small-signal conductance as a function of frequency $J_{0}=50 \mathrm{~A} / \mathrm{cm}^{2}$. (1) Saturated velocity throughout the drift region. (2) Unsaturated velocity in the low-field region[4]. (3) Unsaturated velocity in the low-field region including space-charge conductivity [4]. (4) Numerical results. 


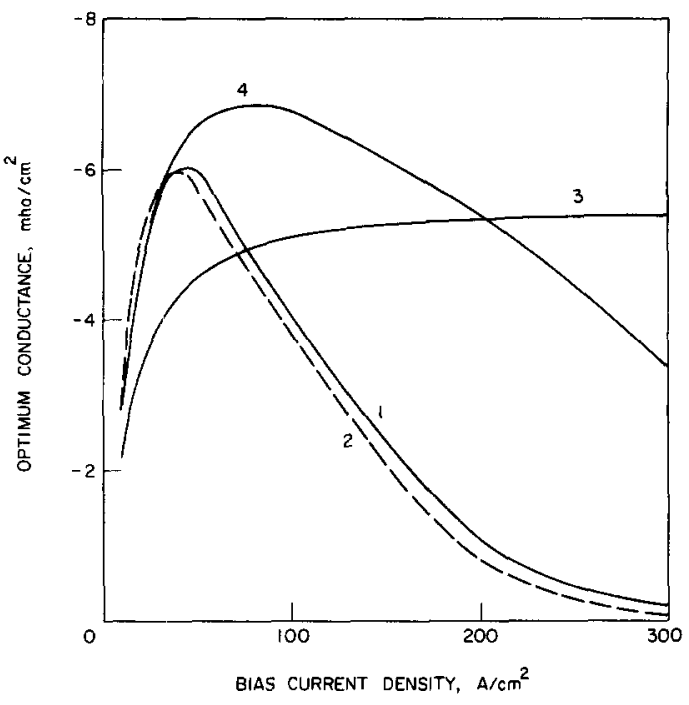

Fig. 3. Optimum small-signal conductance as a function of bias current density. (1) Saturated velocity throughout the drift region. (2) Unsaturated velocity in the low-field region [4]. (3) Unsaturated velocity in the low-field region including space-charge conductivity [4]. (4) Numerical results.

At a low current density $\sigma_{t h}$, being small, dominates and at a moderate or high current density $\sigma_{s c}$ does. The small value of injection conductivity is reflected in the smaller value of negative conductance and a lower operating frequency as shown in curve 3 of Fig. 2. The independence of $\sigma$ on the bias current density at large current densities is also reflected in the constant value of the optimum negative conductance at those current densities (Fig. 3). The decrease of optimum negative conductance of versions one and two at a large current density is due to the increased value of $\sigma_{t h}$. Hence, it can be concluded that the injection conductivity plays an essential role in determining the negative conductance and the operating frequency of the device.

The numerically calculated negative conductance and optimum negative conductance of the $p^{+}-n-p^{+}$structure with a doping profile shown in Fig. 4 are also plotted as curve 4 in Figs. 2 and 3. Thus it can be concluded that the constant saturated drift velocity assumption could, at least in the case of a Si $p-n-p$ structure, lead to an excessively high device operating frequency; also the negative conduc-

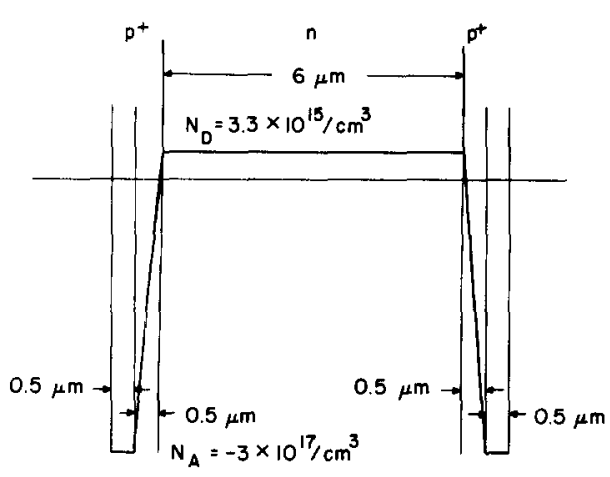

Fig. 4. Doping profile of a $p^{+}-n-p^{+}$structure. tance is sensitive to the model of current injection adopted. The low frequency predicted numerically is partly attributed to the slow carrier motion in the low field region (as can be seen in the large-signal simulation discussed later) and is consistent with the findings of a large phase delay reported elsewhere $[11,12]$.

\section{Approximate large-signal analysis}

The approximate large-signal analysis developed here is analogous to the work of Read[13]. The main distinction here is the different mechanisms of current injection. The thermionic current injection over the barrier $V_{b}(t)$ shown in Fig. 5 is given by $[14,15]$

$$
J_{p}(t)=A^{*} T^{2} \exp \left(-\frac{q}{k T} V_{b}(t)\right)
$$

where

$$
V_{b}(t)=\frac{\epsilon}{2 q N_{D}} E_{0}(t)^{2}
$$

and $E_{0}(t)$ is the electric field at the metallurgical junction. If $V(t)$ represents the voltage drop across the device terminals and $V_{0}$, the dc voltage, then

$$
E_{0}(t)=E_{0 d c}+\frac{V(t)-V_{0}}{w}-\Delta E(t)
$$

where $E_{0 d c}$ is the quiescent value of $E_{0}(t)$ and $\Delta E(t)$ is the induced space-charge electric field which is given by

$$
\Delta E(t)=\frac{1}{w} \int_{0}^{w}(w-x) \frac{\partial E}{\partial x} \mathrm{~d} x .
$$

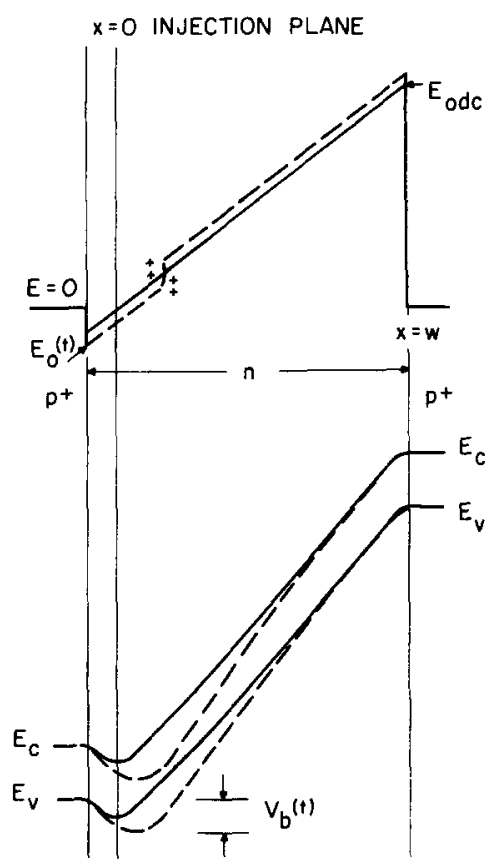

Fig. 5. (a) Electric field profile including the space-charge-induced field of a $p^{+}-n-p^{+}$structure and (b) the corresponding band structure. 
It is clear that the effect of space charge is to make $E_{0}(t)$ more retarding. Thus the presence of mobile space charge tends to quench the injection of carriers.

If hole carriers drift at the saturated velocity, the hole current has the following functional form:

$$
J_{p}(x, t)=J_{p}\left(t-\frac{x}{v_{s}}\right)
$$

The induced space-charge field becomes

$$
\Delta E(t)=\frac{1}{\epsilon \tau} \int_{t-\tau}^{t}\left[\tau-\left(t-t^{\prime}\right)\right] J_{p}\left(t^{\prime}\right) \mathrm{d} t^{\prime}
$$

where the transit time $\tau=w / v_{s}$. Substituting eqns (8) and (9) into eqn (7) yields the following expression for the injection current density:

$$
J_{p}=A^{*} T\left[-\frac{\epsilon}{2 k T N_{D}}\left(E_{0 d c}+\frac{V_{R F}(t)}{w}-\Delta E(t)\right)\right],
$$

where $V_{R F}(t)=V(t)-V_{0}$ is the $R F$ voltage. When eqn (13) is substituted in eqn (12) an integral equation is obtained which can be solved by Picard's iteration method [16].

\section{Results of the approximate large-signal analysis}

The following calculations were made on the structure shown in Fig. 4 except the two $p^{+}-n$ junctions were assumed to be of an abrupt type. The hole saturated velocity was taken to be $10^{7} \mathrm{~cm} / \mathrm{s}$.

The injected hole currents for $V_{R F}$ equal to 5 and $10 \mathrm{~V}$ are given in Fig. 6. The normalized $R F$ voltage $\left(V_{R F} / V_{d c}\right)$ applied across the device is also given in the same figure. Due to space-charge suppression, the phase of the injected current peak has been advanced slightly relative to the maximum voltage point. As the $R F$ voltage becomes larger the injected current behaves more as an impulse of current near the voltage maximum. The induced currents for $V_{R F}=5 \mathrm{~V}$ at various frequencies are given in Fig. 7 . The large-signal admittance of the device biased at $J_{0}=$ $50 \mathrm{~A} / \mathrm{cm}^{2}$ is given in Fig. 8. As expected, the maximum magnitude of the negative conductance occurs at the transit phase angle of approximately $3 \pi / 2$ which occurs at a frequency near $13 \mathrm{GHz}$.

The injected current resembles an impulse in phase with the $R F$ voltage. When the $R F$ voltage amplitude is large the maximum efficiency of the device can be shown to be

$$
\eta_{\max }=\frac{2}{3 \pi}
$$

where $V_{R F}$ has been set equal to the d.c. voltage $V_{0}$. In order to estimate the power capability of the device it is necessary to know the current handling capability. Incorporating (1) the induced electric field by the moving space charge as shown in Fig. 9, (2) the large-signal constraint of avalanche breakdown and (3) the requirement of minimum electric field needed for carriers moving at the saturated

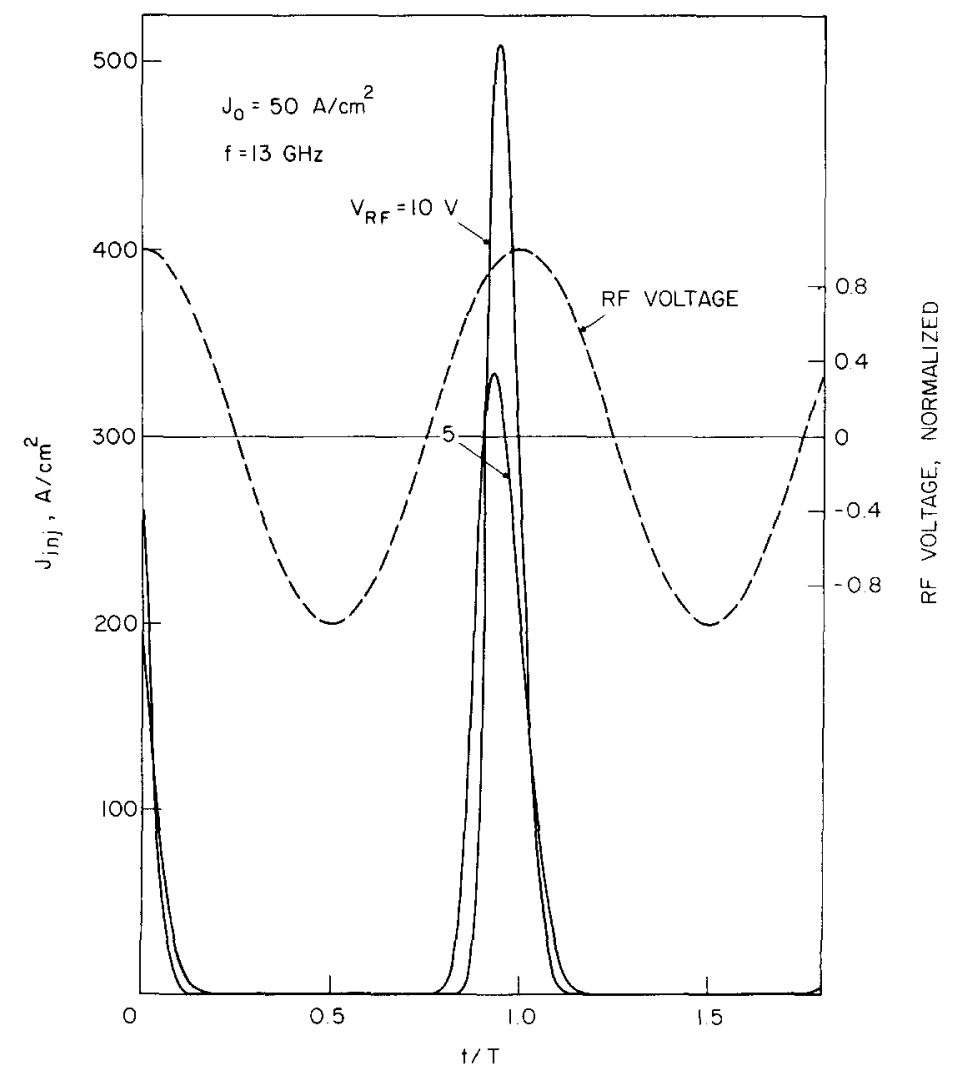

Fig. 6. Injected current density and applied $R F$ voltage. 


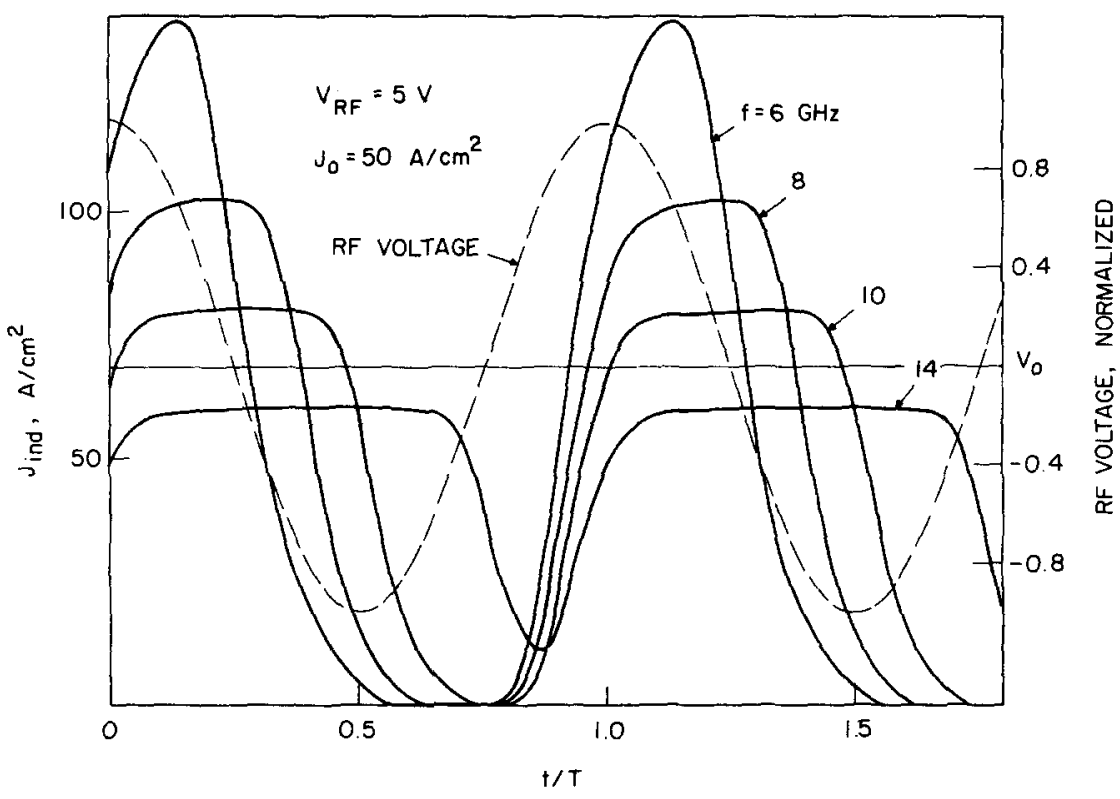

Fig. 7. Induced particle current density at various frequencies.

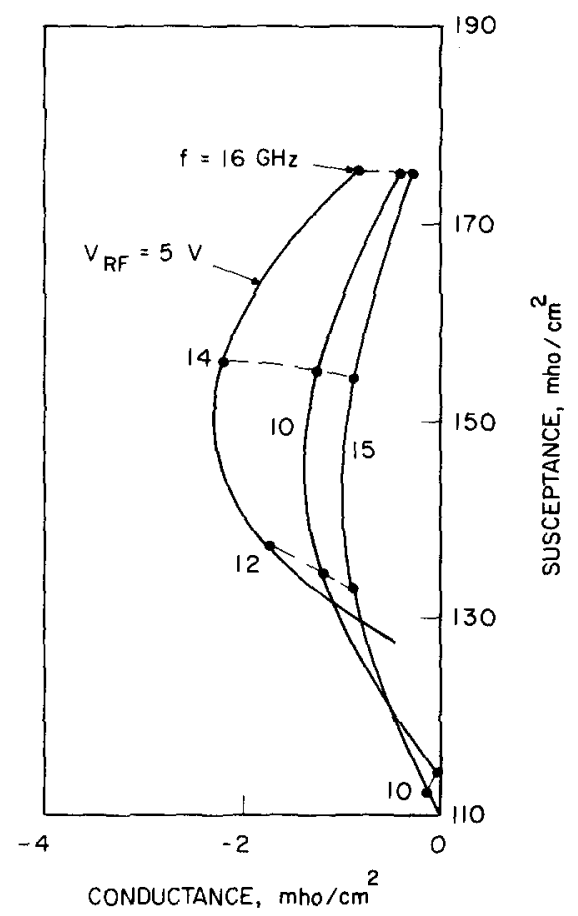

Fig. 8. First-order large-signal admittance of a $p^{+}-n-p^{+}$structure $J_{0}=50 \mathrm{~A} / \mathrm{cm}^{2}$.

velocity, Rüegg[1] showed that the optimum bias current density is

$$
J_{0}=\frac{3 \epsilon f_{0} E_{m}}{2},
$$

where $f_{0}=$ the operating frequency,

$\epsilon=$ the dielectric constant and

$E_{m}=$ the avalanche breakdown field.

For a Si device operated at $10 \mathrm{GHz} J_{0} \sim 3000 \mathrm{~A} / \mathrm{cm}^{2}$, where $E_{m}=2 \times 10^{5} \mathrm{~V} / \mathrm{cm}$ has been used. The corresponding power density is

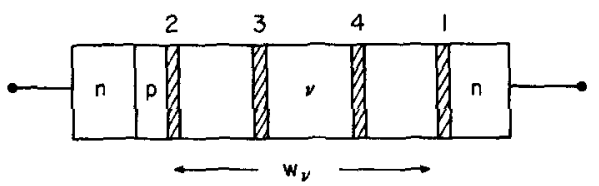

(a)

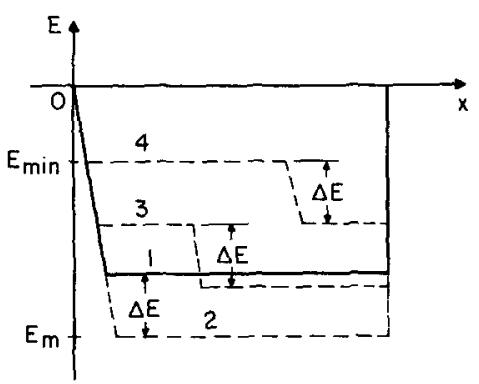

(b)

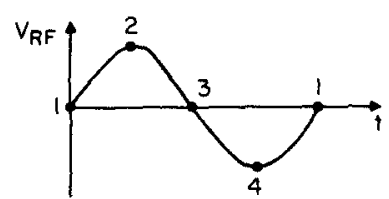

(c)

Fig. 9. (a) $n-p-\nu-n$ structure, (b) electric field profile at four $R F$ phases and (c) $R F$ voltage vs time.

$$
P_{\max }=\frac{3 v_{s}}{16 \pi} \epsilon E_{m}^{2}
$$

which is approximately equal to $24,000 \mathrm{~W} / \mathrm{cm}^{2}$, where $v_{s}=1 \times 10^{7} \mathrm{~cm} / \mathrm{s}$ has been used. As is seen later, both the efficiency and optimum current density predicted from this approximate analysis are one order of magnitude larger than the experimental and numerically obtained values. 
Thus, the predicted $R F$ power is approximately two orders of magnitude higher than can actually be achieved [2-4]. Therefore, the approximate analysis cannot be employed to determine the capabilities of this device realistically.

\section{NUMERICAL SIMULATION OF A Si $n-p-\nu-n$ STRUCTURE}

A computer simulation of a Si $n-p-\nu-n$ structure was carried out. The device considered is device 3 of Table 1 where the parameters are given. Due to computational cost, nondegenerate doping densities are assigned to the two end regions. However, as long as the values are sufficiently high, the device performance is not expected to be greatly affected by this limitation. The device is operated near its optimum power point, where the bias current density is $J_{0}=180 \mathrm{~A} / \mathrm{cm}^{2}$, the $R F$ voltage amplitude is $V_{R F}=60 \mathrm{~V}$, the quiescent voltage is $V_{0}=109.6 \mathrm{~V}$ and the frequency of operation is $f=10.5 \mathrm{GHz}$.

The injected particle current distribution at eight different phases of the $R F$ voltage cycle is shown in Fig. 10(a) where the phases are indicated by:

\begin{tabular}{|c|c|}
\hline Label & Phase angle (degrees) \\
\hline 1 & 269.5 \\
\hline 2 & 314.8 \\
\hline 3 & 360.0 \\
\hline 4 & 43.4 \\
\hline 5 & 88.6 \\
\hline 6 & 133.9 \\
\hline 7 & 179.1 \\
\hline 8 & 224.3 \\
\hline
\end{tabular}

The corresponding electric field profile and carrier density distribution are given, respectively, in Fig. 10(b) and (c). The induced current and the applied $R F$ voltage are shown in Fig. 10(d). At phase 1 the voltage across the device is near its maximum value and the retarding electric field at the left-hand-side (LHS) junction has diminished to a small value. Particle current and a large number of minority carriers are injected on the LHS. Meanwhile most of the previously injected carriers have been collected on the RHS as shown in Fig. 10(c). At phase 3 the voltage drops to its quiescent value and the injected carriers drift to nearly the middle of the depletion region. Then at phase 5 the voltage across the device is near its minimum, the magnitude of the retarding electric field at the LHS junction is near its maximum and those carriers that were injected earlier had drifted to the RHS more than halfway across the depletion region. Carriers in the leading edge of the packet are being collected. At phase 7 the voltage returns to its quiescent value. The peak of the drifting carrier packet has now reached the collector. Meanwhile, the magnitude of the retarding field has also been reduced. The $R F$ cycle then repeats itself at each microwave period.

There are several basic mechanisms which are revealed in the preceding simulation:

(a) As assumed in the commonly accepted theory the

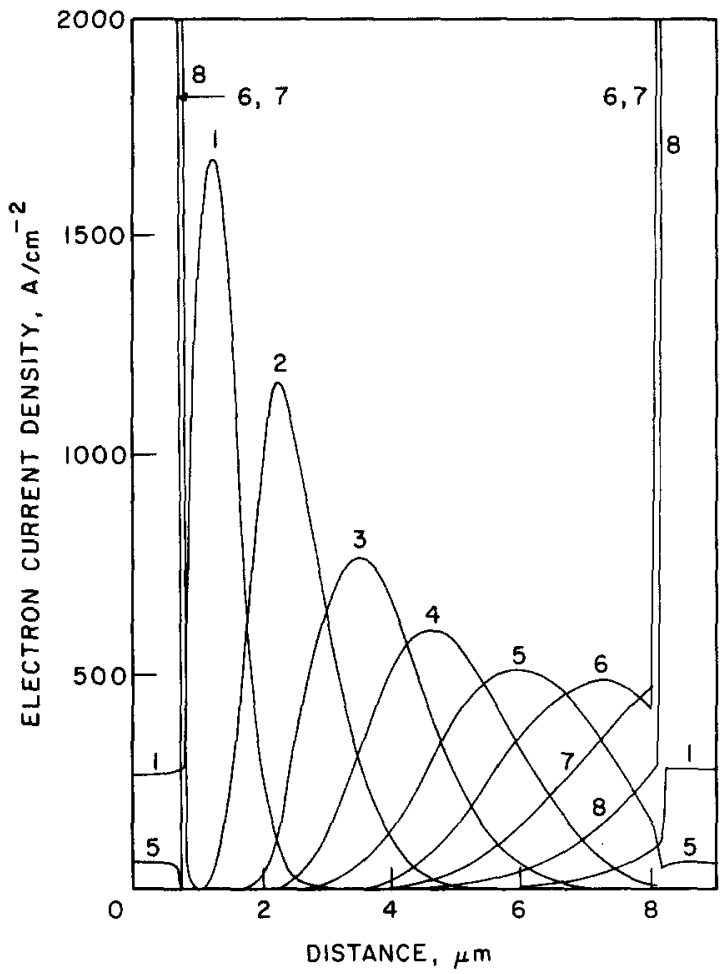

(a)

Table 1. Parameters of X-band BARITT devices considered

\begin{tabular}{|c|c|c|c|c|c|c|c|c|c|c|c|}
\hline Device No. & Material & Structure & $\begin{array}{c}\mathrm{x}_{\mathrm{Al}} \\
\left(\mathrm{cm}^{-3}\right) \\
\end{array}$ & $\begin{array}{c}\mathrm{N}_{\mathrm{D}} \\
\left(\mathrm{cm}^{-3}\right) \\
\end{array}$ & $\begin{array}{c}\mathbb{N}_{v} \\
\left(\mathrm{~cm}^{-3}\right) \\
\end{array}$ & $\begin{array}{r}\mathrm{N}_{\mathrm{A} 2} \\
\left(\mathrm{~cm}^{-3}\right) \\
\end{array}$ & $\begin{array}{c}w_{D} \\
(\mu m) \\
\end{array}$ & 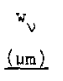 & $\begin{array}{r}w_{T L} \\
(\mu m) \\
\end{array}$ & $\begin{array}{r}w_{T R} \\
(\mu m) \\
\end{array}$ & $\begin{array}{l}w_{p} \\
\underline{(u m)} \\
\end{array}$ \\
\hline .2 & $\mathrm{Si}$ & $p-n-p$ & $3 \times 10^{17}$ & $3.3 \times 10^{15}$ & -- & $3 \times 10^{17}$ & 6.0 & - & 0.5 & 0.5 & 0.5 \\
\hline 2 & si & $n-p-n$ & $3 \times 10^{17}$ & $3.3 \times 10^{15}$ & -- & $3 \times 10^{27}$ & 6.0 & -- & 0.5 & 0.5 & 0.5 \\
\hline 3 & $\mathrm{Si}$ & $n-p-v-n$ & $5 \times 10^{17}$ & $2.0 \times 10^{16}$ & $2 \times 10^{13}$ & $2 \times 10^{17}$ & 0.7 & 6.3 & 0.7 & 0.5 & 0.3 \\
\hline 4 & GaAs & $n-p-n$ & $5 \times 10^{16}$ & $2.0 \times 10^{15}$ & -- & $5 \times 10^{16}$ & 7.0 & - & 0.5 & 0.5 & 0.5 \\
\hline
\end{tabular}

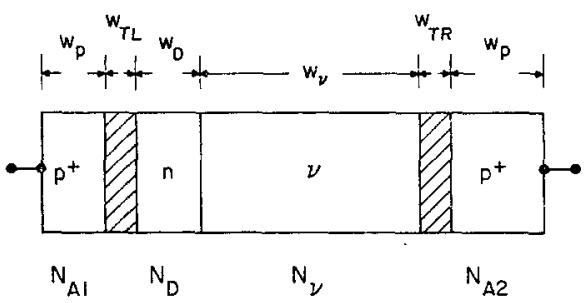




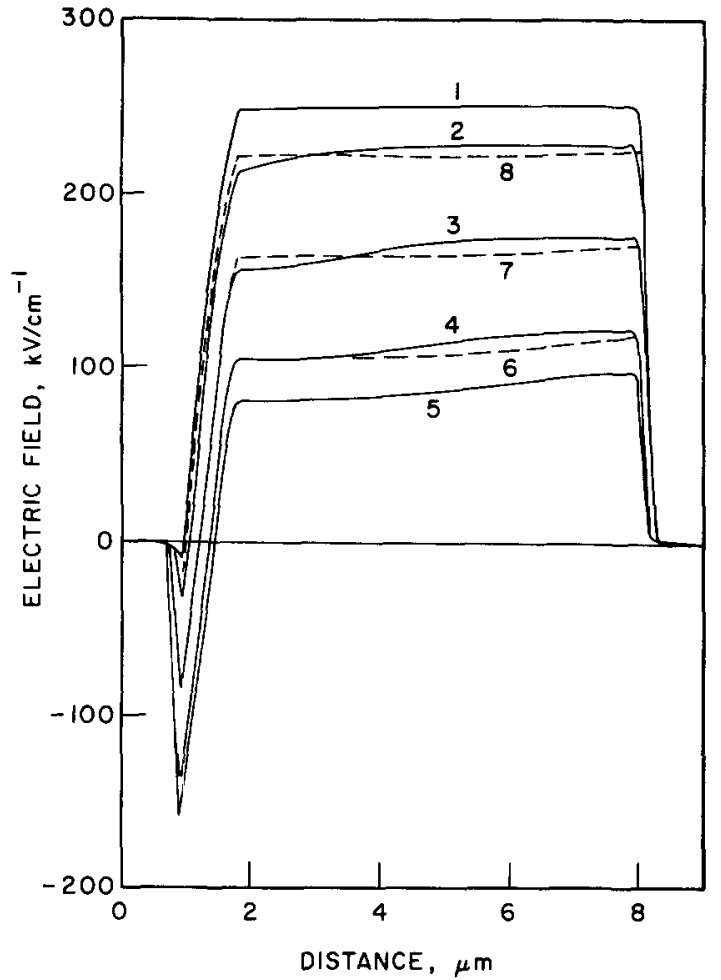

(b)

Fig. 10(b).

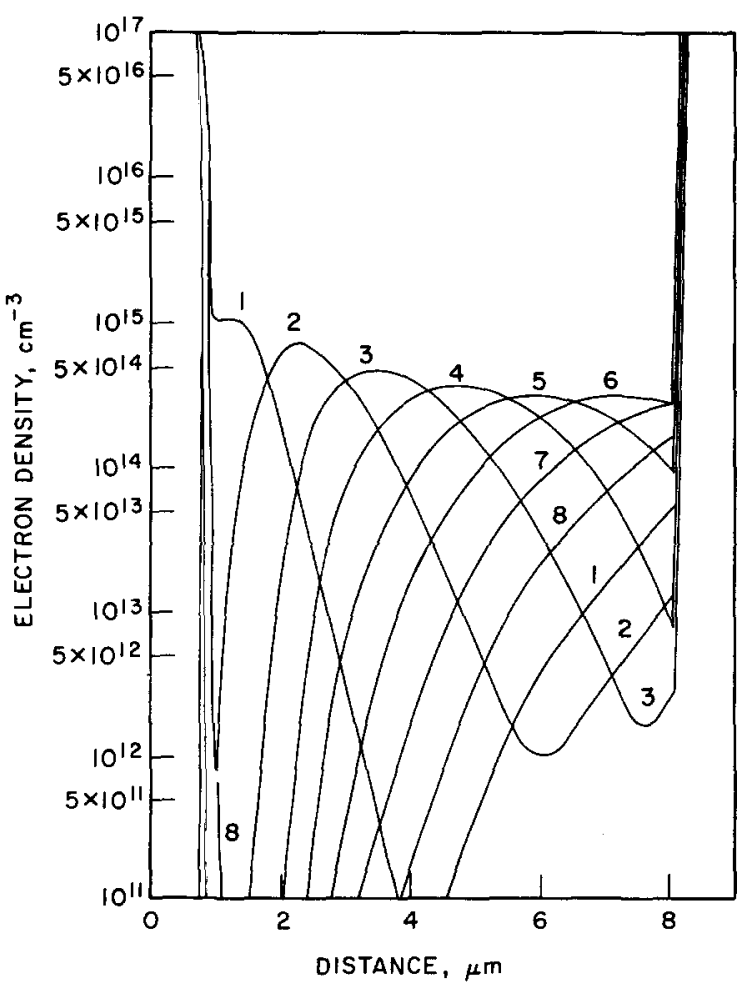

(c)

Fig. 10(c).

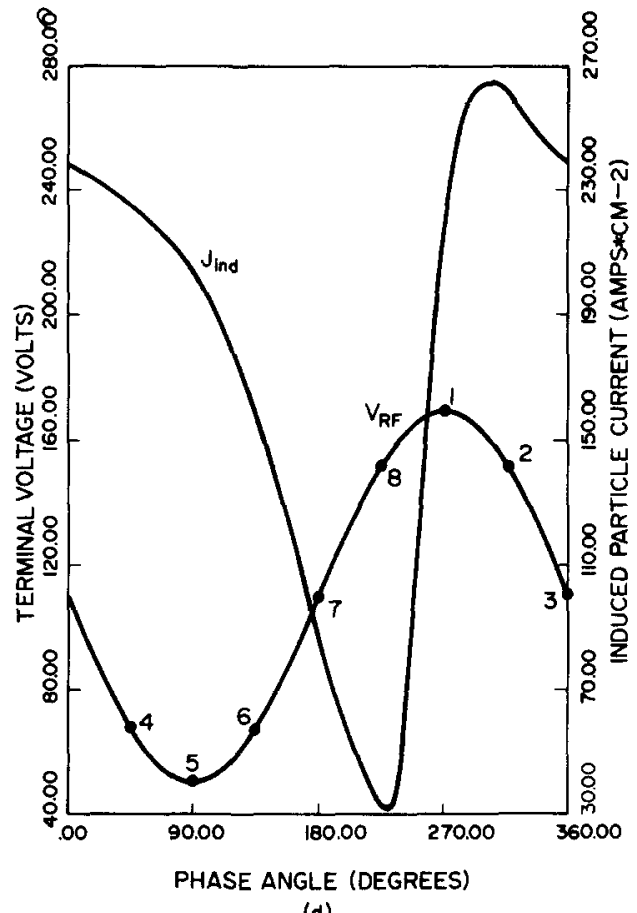

(d)

Fig. 10(d).

Fig. 10a-d. Injected particle current, electric field and electron density at various phases of the $R F$ voltage, and the $R F$ voltage and induced current density.

current injection does take place in phase with the electric field and hence the voltage across the device.

(b) Unlike the d.c. case or a very slowly time-varying case, the electric field profile does not collapse in such a way that the device becomes unpunched through even when the terminal voltage falls below the punch-through value. Provided recombination and generation lifetimes are much larger than the microwave period, the device once biased at punch through remains depleted of majority carriers throughout the whole cycle of the microwave period.

(c) The electric field profile varies in phase with the terminal voltage. Even under the large-signal optimum power condition, the injected minority carrier space charge remains moderately low compared to the impurity level, hence the whole structure acts largely as if it were a dielectric capacitor. Thus the device $Q$ factor is expected to be large.

(d) Even in the Read structure where the low-field region has been minimized, significant carrier-packet dispersion still occurs. Two basic mechanisms are responsible for such dispersion. One is the spatial velocity modulation arising from the nonuniformity of the electric field causing carriers at different positions to move with different velocities. Thus, depending on the electric field profile carriers may be bunched together or debunched. Unfortunately, at least in the case at hand (Fig. 10) the monotonically increasing electric field in the low-field drift region of the BARITT device tends to debunch the carriers resulting in packet broadening. Without carrier diffusion the magnitude of the carrier packet however broadened by velocity modulation would remain undiminished. The other 
mechanism is carrier diffusion arising from the presence of a concentration gradient. As long as such concentration gradient exists, whether it exists at the outskirt or at the equalize the distribution at the expense of high carrier concentration.

By referring to Fig. 10a-c it can be seen that, during the interval between phase 1 and phase 2, the injected carriers are clustered in the region $x=(1,1.8 \mu \mathrm{m})$, where the electric field is rapidly increasing from a very small value to near its velocity saturation value. Hence, the spatial velocity modulation $\Delta v$ arising from it is nearly $10^{7} \mathrm{~cm} / \mathrm{s}$. On the other hand the carrier diffusion velocity, which can be estimated to be

$$
v_{\text {diff }}=D(E)(\Delta n / n)(1 / \Delta x)
$$

is of the order of $10^{6} \mathrm{~cm} / \mathrm{s}$. The concentration gradient at $x \approx 1 \mu \mathrm{m}$ was used for the calculation. Although significant packet broadening can be attributed to velocity modulation the large reduction in the magnitude of carriers and especially the current packet is due to diffusion. majority of the injected carriers are confined in the region $x=(1.8,7.5 \mu \mathrm{m})$. The electric field arising from the doping impurities is relatively flat. The spatial velocity modulation arises mainly from the space-charge field induced by the injected carriers. This is found to be negligible. The following table lists the values of velocity modulation taken as the difference between the drift velocity at the peak of the carrier packet and that at the half value point to the left of the peak. center of the carrier packet, they will disperse so as to

During the interval between phase 2 and phase 5 the

As expected the most significant carrier dispersion occurs at phase 2 where the diffusion velocity assumes its maximum value of $2.8 \times 10^{5} \mathrm{~cm} / \mathrm{s}$ and it gradually $\mathrm{di}$ minishes as the diffusion velocity decreases between phases 3 and 5 .

(e) The injected carriers move with different velocities during different phases of the $R F$ cycle. Under a large $R F$ voltage amplitude the modulation of the electric field can be sufficiently large that the minimum electric field strength is excessively depressed causing the carriers to drift at a lower velocity and in the extreme case to significantly diffuse contributing to reduction of current packet magnitude and broadening of its width prior to the collection of carriers. The locus of the peak of the current packet as a function of the phase angle of the $R F$ cycle is plotted in Fig. 11. The slope of such a curve is proportional to the packet velocity. Its values at three different phase angles are listed below:

\begin{tabular}{cc} 
Phase angle (degrees) & Packet velocity $(\mathrm{cm} / \mathrm{s})$ \\
\hline 270 & $1.47 \times 10^{6}$ \\
360 & $1.05 \times 10^{7}$ \\
$90(+360)$ & $6.50 \times 10^{6}$
\end{tabular}

At 270 degrees the $R F$ voltage is maximum and carriers are injected into the low-field region where they move slowly. At 360 degrees the injected carriers have moved to the middle of the device, the electric field value is approximately $170 \mathrm{kV} / \mathrm{cm}$ and the packet moves at the saturated velocity. At $90(+360)$ degrees the $R F$ voltage is at its minimum, the electric field level is depressed to approximately $80 \mathrm{kV} / \mathrm{cm}$ and the velocity is reduced to $6.5 \times$ $10^{6} \mathrm{~cm} / \mathrm{s}$. The case discussed above corresponds to the

\begin{tabular}{cccccc} 
Phase & $\begin{array}{c}E_{\min } \\
(\mathrm{kV} / \mathrm{cm})\end{array}$ & $\begin{array}{c}E_{\max } \\
(\mathrm{kV} / \mathrm{cm})\end{array}$ & $\begin{array}{c}v_{\min } \\
(\mathrm{cm} / \mathrm{s})\end{array}$ & $\begin{array}{c}v_{\max } \\
(\mathrm{cm} / \mathrm{s})\end{array}$ & $\begin{array}{c}\Delta v \\
(\mathrm{~cm} / \mathrm{s})\end{array}$ \\
\hline 2 & 213.6 & 220.1 & $1.0025 \times 10^{7}$ & $1.0035 \times 10^{7}$ & $1 \times 10^{4}$ \\
3 & 158.1 & 164.7 & $0.9899 \times 10^{7}$ & $0.9918 \times 10^{7}$ & $1.2 \times 10^{4}$ \\
4 & 107.6 & 112.5 & $0.9680 \times 10^{7}$ & $0.9709 \times 10^{7}$ & $2.9 \times 10^{4}$ \\
5 & 84.8 & 90.0 & $0.9503 \times 10^{7}$ & $0.9551 \times 10^{7}$ & $4.8 \times 10^{4}$
\end{tabular}

Clearly, velocity modulation plays little role in the carrier dispersion. This point is best illustrated by the relatively constant carrier packet between phases 5 and 6 while the velocity modulation assumes its maximum value of $4.8 \times$ $10^{4} \mathrm{~cm} / \mathrm{s}$ at phase 5 . The appreciable packet broadening and reduction of its magnitude between phases 2 and 5 must be attributed to diffusion. The large carrier-concentration gradient at the LHS outer skirt of the packet between phases 2 and 4 is responsible for the eventual packet dispersion. To verify this point the diffusion velocities associated with concentration fall off from $10^{13} / \mathrm{cm}^{3}$ to $10^{12} / \mathrm{cm}^{3}$ at the four phases are calculated and listed in the following table:

\begin{tabular}{ccccc} 
Phase & $\begin{array}{c}\Delta x \\
(\mu \mathrm{m})\end{array}$ & $\begin{array}{c}E_{\max } \\
(\mathrm{kV} / \mathrm{cm})\end{array}$ & $\begin{array}{c}D\left(E_{\max }\right) \\
\left(\mathrm{cm}^{2} / \mathrm{s}\right)\end{array}$ & $\begin{array}{c}v_{\text {diff }} \\
(\mathrm{cm} / \mathrm{s})\end{array}$ \\
\hline 2 & 0.13 & 55 & 4.1 & $2.8 \times 10^{5}$ \\
3 & 0.20 & 157 & 1.6 & $7.2 \times 10^{4}$ \\
4 & 0.28 & 103 & 2.3 & $7.4 \times 10^{4}$ \\
5 & 0.42 & 83 & 2.9 & $6.2 \times 10^{4}$
\end{tabular}

optimum $R F$ voltage value for which the power is maximum. For an $R F$ voltage value larger than this optimum value the electric field at the minimum voltage phase falls even below $80 \mathrm{kV} / \mathrm{cm}$ and thus further reduces the peak value of the current packet and broadens its width, meanwhile slowing down the packet velocity. This large signal field depression is mainly responsible for the power and efficiency degradation at large-signal values above the optimum one as shown in Fig. 12. This power degradation constitutes the large-signal power limitation which was neglected in the first-order theory. The slow packet velocity at the low-field region and the large-signal field depression, to a lesser extent, could contribute to a significantly lower operating frequency of the device (e.g., Fig. 2). For the Read structure of Fig. 10, the discrepancy becomes insignificant because of the narrow low-field region.

Power capability of the BARITT device

In order to estimate the power capability of BARITT 


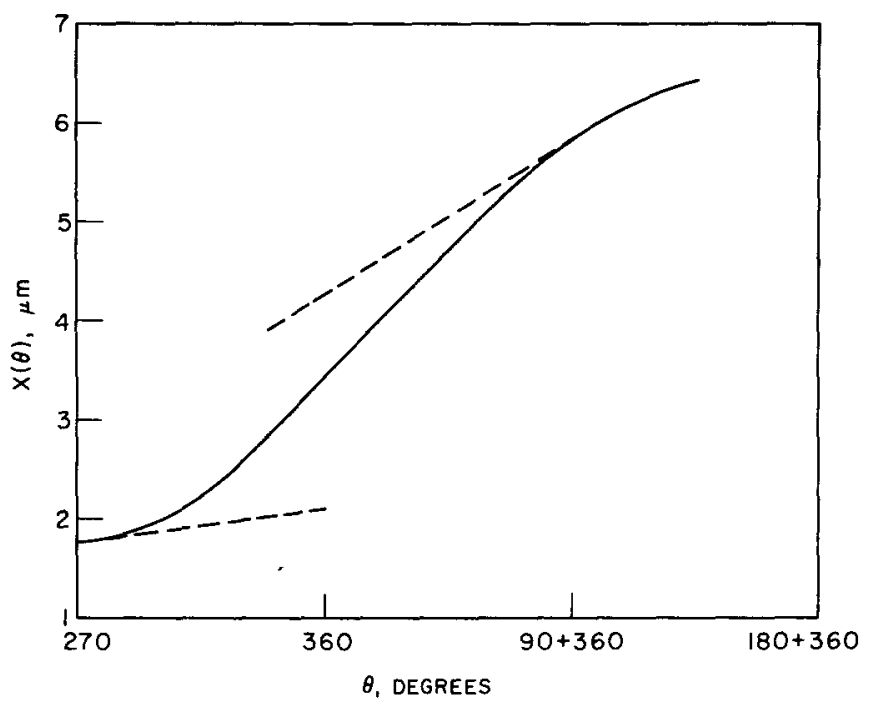

Fig. 11. Locus of the peak of the injected current packet.

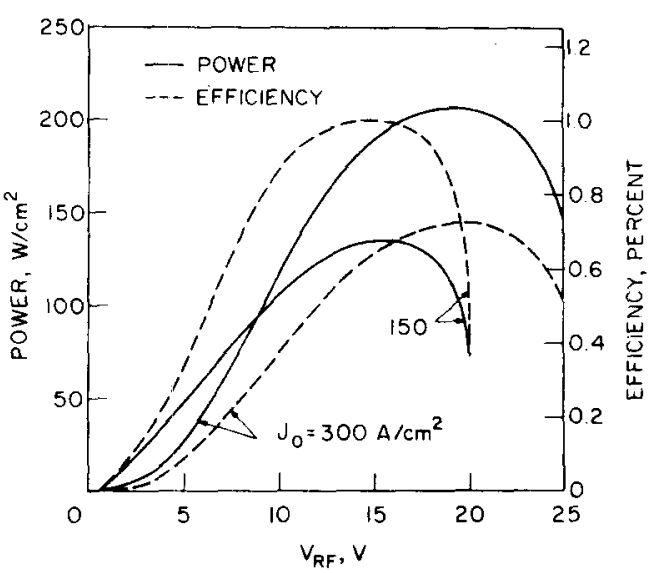

Fig. 12. Power output and efficiency vs $R F$ voltage for device 1 .

devices four X-band devices consisting of Si $p-n-p, n-p-n$ and $n-p-\nu-n$ and GaAs $n-p-n$ structures were analyzed numerically [4]. For a given width and d.c. current density the impurity doping density of the uniformly doped structure is maximized, while for the Read structure that of the narrow and heavily doped region is maximized. In both cases the limitation of avalanche breakdown is imposed.

The calculation involves solving the d.c. continuity equations and Poisson's equation:

and

$$
\begin{aligned}
-\frac{1}{q} \frac{\partial J_{p}}{\partial x}+\alpha_{n} J_{n}+\alpha_{p} J_{p} & =0, \\
\frac{1}{q} \frac{\partial J_{n}}{\partial x}+\alpha_{n} J_{n}+\alpha_{p} J_{p} & =0
\end{aligned}
$$

$$
\frac{\partial E}{\partial x}=\frac{q}{\epsilon}\left(N_{D}+p\right)
$$

where

$$
\begin{aligned}
& J_{p}=q \mu_{p}\left(p E-\frac{k T}{q} \frac{\partial p}{\partial x}\right), \\
& J_{n}=q \mu_{n}\left(n E-\frac{k T}{q} \frac{\partial n}{\partial x}\right) .
\end{aligned}
$$

For simplicity it is assumed that the electric field at the LHS junction is zero as shown by the dashed line in Fig. 13 and the carrier space charge is given by

$$
p=\frac{J_{p}}{q\langle v\rangle}
$$

where $\langle v\rangle$ is an average velocity of carriers. If the avalanche region is confined to be the region between $x_{1}$ and $w$, which can be readily determined by excluding the low-field region whose contribution to the ionization integral is negligible (in this case less than 0.05), then minority carriers injected from the LHS barrier potential are multiplied in the multiplication region $\left[x_{1}, w\right]$. The current boundary conditions are:

$$
\begin{aligned}
& J_{n}\left(x_{1}\right)=J_{0}-J_{p}\left(x_{1}\right), J_{n}(w)=J_{n s}, \\
& J_{p}\left(x_{1}\right)=J_{p}\left(x_{1}\right), J_{p}(w)=J_{0}-J_{n s} .
\end{aligned}
$$

If it is assumed that the minority-carrier injection is not affected by the majority carriers generated by the avalanche process, then the following expression can be derived from the hole current component of eqn (17) and the boundary conditions of eqn (21)

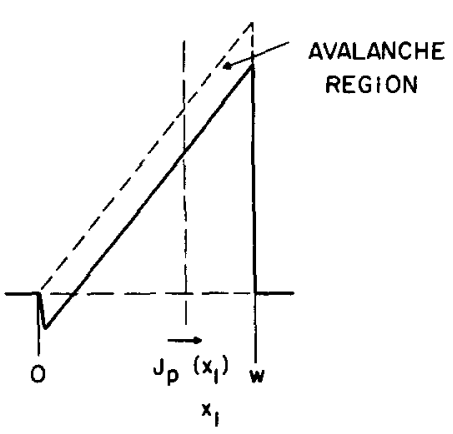

Fig. 13. Avalanche region in a BARITT structure. 


$$
J_{0}=J_{p}\left(x_{1}\right)\left[\frac{\exp \left[-\int_{x_{1}}^{w}\left(\alpha_{n}-\alpha_{p}\right) \mathrm{d} x\right]}{1-\int_{x_{1}}^{w} \alpha_{n} \exp \left[-\int_{x}^{w}\left(\alpha_{n}-\alpha_{p}\right) \mathrm{d} x^{\prime}\right] \mathrm{d} x}\right] .
$$

The term in the large brackets represents the amount of multiplication the minority carriers undergo. The condition for the initiation of avalanche breakdown has been set rather arbitrarily to be

$$
\frac{\exp \left(-\int_{x_{1}}^{w}\left(\alpha_{n}-\alpha_{p}\right) \mathrm{d} x\right)}{1-\int_{x_{1}}^{w} \alpha_{n} \exp \left(-\int_{x}^{w}\left(\alpha_{n}-\alpha_{p}\right) \mathrm{d} x^{\prime}\right) \mathrm{d} x}=2,
$$

where

$$
\begin{aligned}
& \alpha_{n}=3.8 \times 10^{6} \exp \left(-\frac{1.75 \times 10^{6}}{E}\right) \mathrm{cm}^{-1} \\
& \alpha_{p}=2.25 \times 10^{7} \exp \left(-\frac{3.26 \times 10^{6}}{E}\right) \mathrm{cm}^{-1}
\end{aligned}
$$

for $\mathrm{Si}[17]$, and

$$
\alpha_{n}=\alpha_{p}=2 \times 10^{5} \exp \left[-\left(\frac{5.5 \times 10^{5}}{E}\right)^{2}\right] \mathrm{cm}^{-1}
$$

for GaAs[14] where the electric field is in $V / \mathrm{cm}$.

The four devices, whose parameters are given in Table 1, were chosen under the constraint of avalanche breakdown at a current density of $1000 \mathrm{~A} / \mathrm{cm}^{2}$ which is much higher than the device operating current density. In all of the cases considered here it was found that the maximum electric field, even under the optimum large-signal condition for maximum power, never exceeds the value determined by eqns (17-20). Thus the nonoccurrence of avalanche breakdown is assured. A summary of the properties of these devices is given in Table 2. For the Si devices considered here and designed to operate in the $\mathrm{X}$-band frequency range the expected power and efficiency are approximately $300 \mathrm{~W} / \mathrm{cm}^{2}$ and $3 \%$, respectively. When compared to the values of $24,000 \mathrm{~W} / \mathrm{cm}^{2}$ and $21 \%$ predicted by the firstorder theory the actual power is approximately two orders of magnitude smaller and the efficiency, one order of magnitude. It is worth noting however that improved efficiency and power output may be obtained with more complicated doping profiles.

\section{Power limitations}

The power generated by a BARITT device is determined by the time average of the product of the induced current and the $R F$ voltage in a microwave period [see Fig. 10(d)]. There are four factors that determine the device power at a particular current level. These are:

1. The peak value of the induced current.

2. The shape of the induced current.

3. The magnitude of the $R F$ voltage.

4. The frequency of operation.

The space-charge-limited current injection becomes dominant at large current levels; it thus sets a limit to the peak value of the induced current. Aside from current injection and collection, the dispersion of the injected current packet (magnitude reduction and packet broadening) during the transit time largely determines the shape of the induced current waveform. Power (and efficiency) actually decrease when the amplitude of the $R F$ voltage exceeds an optimum value (Fig. 12) thus resulting in a large-signal power limitation. The frequency of operation becomes important due to the well-known $P f^{2}$ law of transit-time devices.

In order to account for the large discrepancy between

\begin{tabular}{|c|c|c|c|c|}
\hline Device & 1 & 2 & 3 & 4 \\
\hline & Si $p-n-p$ & Si $n-p-n$ & $\underline{B i-n-p-v-n}$ & GaAs $n-p-n$ \\
\hline \multicolumn{5}{|c|}{ (a) Meximun Power } \\
\hline Power $\left(\mathrm{W} / \mathrm{cm}^{2}\right)$ & 257 & 230 & 260 & 25 \\
\hline Efficiency (percent) & 1.10 & 1.47 & 1.37 & 1.03 \\
\hline Frequency $(\mathrm{GHz})$ & 9.5 & 11.5 & 10.5 & 9.0 \\
\hline $\mathrm{J}_{0}\left(\mathrm{~A} / \mathrm{cm}^{2}\right)$ & 260 & 205 & 175 & 40 \\
\hline$v_{0}(\mathrm{v})$ & 89.4 & 76.4 & 209 & 60.6 \\
\hline $\mathrm{V}_{\mathrm{RF}}(\mathrm{v})$ & 25 & 30 & 60 & 30 \\
\hline $\mathrm{v}_{\mathrm{BF}} / \mathrm{v}_{0}$ & 0.28 & 0.39 & 0.55 & 0.50 \\
\hline \multicolumn{5}{|c|}{ (b) Maximum Efficiency } \\
\hline Efficiency (percent) & 2.28 & 2.63 & 2.5 & 1.3 \\
\hline Power $\left(\mathrm{w} / \mathrm{cm}^{2}\right)$ & 210 & 103 & 180 & 20 \\
\hline Frequency (GHz) & 9.0 & 11.5 & 10.2 & 9.0 \\
\hline$J_{0}\left(A / \mathrm{cm}^{2}\right)$ & 110 & 53 & 65 & 27 \\
\hline$v_{a}(v)$ & 83.5 & 73.4 & 111 & 57.0 \\
\hline $\mathrm{v}_{\mathrm{RF}}(\mathrm{V})$ & 22 & 25 & 55 & 30 \\
\hline$v_{R F} / v_{0}$ & 0.26 & 0.34 & 0.50 & 0.53 \\
\hline
\end{tabular}
the first-order theory (FOT) predictions of power and efficiency and that obtained numerically, it is convenient to express the power obtained numerically in terms of the

Table 2. BARITT device capabilities 
product of the value predicted from FOT and the following four power reduction factors: space-charge-limited injection $\left(F_{s c}\right)$, induced current tapering $\left(F_{i c}\right)$, large-signal limitation $\left(F_{i s}\right)$ and frequency reduction $\left(F_{f}\right)$ as

$$
P_{\text {num }}=P_{\text {For }} \cdot F
$$

where

$$
F=F_{s c} \cdot F_{i c} \cdot F_{i s} \cdot F_{f}
$$

Based on the results of the FOT and the numerical computation the four reduction factors can be estimated. The values of pertinent parameters of the Read structure of Table 2 together with their respective power reduction factors are listed in Table 3.

According to the FOT [1] it was assumed that a very large current impulse could be injected under a large-signal condition. Under this condition the induced space-charge field was sufficiently large to excessively depress the electric field profile as shown in Fig. 9. The assumption is in error because even under the optimum large $R F$ signal of $60 \mathrm{~V}$ only a limited amount of carriers $\left(10^{15} / \mathrm{cm}^{3}\right)$ are injected as is evident in Fig. 10(c). This is attributed to space-charge-limited thermionic injection where current, instead of rising exponentially with the square of applied voltage, becomes linearly dependent on the voltage $[4,15]$. This space-charge-limited injection excessively constricts the peak induced current value to merely one tenth of the optimum value predicted by the FOT[1] (Table 3). Incidentally, since carrier mobility, diffusion and doping profile of the injecting junction affect the thermionic potential barrier, they strongly influence the properties of BARITT devices as evident in Table 2 . This subject will be pursued further in later sections.

The tapering off of the induced current waveform can be attributed to velocity modulation, carrier diffusion and carrier collection at various phases of the cycle. Between phases 1 and 2 current packet dispersion due to velocity modulation and carrier diffusion causes the induced current (Fig. 10(d)) to decrease. Between phases 2 and 5 diffusion dominated packet dispersion continues to cause the induced current to diminish. Between phases 5 and 7 the current falls off sharply due to premature collection of the leading portion of the packet. Between phases 7 and 8 the collection of the trailing part of the packet takes place. Since, during the period from phase 1 to phase 3 , the device is dissipating power the reduction of the induced current may be beneficial to power generation. However, the tapering off of the waveform between phases 3 and 5 due to diffusion and between phases 5 and 7 arising from premature collection are detrimental to device power generation. Despite the complexity of the matter it can be concluded that the combined effects of velocity modulation, carrier diffusion and collection of the dispersed carrier packet could significantly reduce the device power; in this case by a factor of five from its value predicted by the FOT. The large-signal depression of the electric field contributes to a reduced value of $R F$ voltage amplitude. In this case power is reduced by a factor of two. Finally, because of the narrow low-field region of the Read structure analyzed no appreciable power degradation is expected due to frequency reduction. However, this factor will be significant for other structures. The reduction factor of efficiency is the product of $F_{s c}, F_{i c}$ and $F_{l s}$ which amounts to a factor of ten as expected.

\section{Effects of doping profile}

It is commonly believed that the Read structure, such as an $n-p-\nu-n$ one, with a heavily doped narrow $p$-region followed by an intrinsic drift region is highly desirable for high-power and/or high-efficiency operation of a BARITT device [1,2]. This is based on the intuitive impression that such a structure provides a fast-rising electric field profile at the injection region which increases the punch-through voltage and also helps sweep away the injected minority carriers rapidly, thereby increasing the device signal handling capability prior to the space-charge domination of carrier injection. This has been found to be true from the results of the numerical calculations shown in Table 2 where the ratio of the optimum $R F$ voltage amplitude to the d.c. voltage is higher for the Read $n-p-\nu-n$ structure than for the uniformly doped $n-p-n$ structure. On the other hand, the magnitude of the negative conductance of the $n-p-\nu-n$ Read structure is smaller than the $n-p-n$ counterpart as shown in the optimum negative conductance plots of Fig. 14. The magnitude of negative conductance of a uniformly doped $\mathrm{Si} p-n-p$ structure is reduced when the impurity doping density is increased from $1.5 \times 10^{15} \mathrm{~cm}^{-3}$ to $1.75 \times 10^{15} \mathrm{~cm}^{-3}$ as shown in Fig. 15. The power output and efficiency values of the $n-p-\nu-n$ and $n-p-n$ structures are comparable.

\section{Effects of material parameters}

The effects of material parameters on the characteristics of a BARITT device can be conveniently categorized into two classes: high-field and low-field effects. The high-field effect is controlled by the saturated velocity of

\begin{tabular}{|c|c|c|c|}
\hline Parameter & FOT_Result & Numerical Result & Feduction Factor \\
\hline $\begin{array}{l}\text { Peak Induced } \\
\text { Current Dersity }\end{array}$ & $\begin{array}{c}3000 \mathrm{~A} / \mathrm{cm}^{2} \\
(\mathrm{Eq} \cdot 15)\end{array}$ & $\begin{array}{c}260 \\
{[F i g .10(a)]}\end{array}$ & $F_{S C}-0.1$ \\
\hline $\begin{array}{l}\text { Optimum } R F \\
\text { Voltage }\end{array}$ & $\begin{array}{c}110 \mathrm{~V} \\
\text { (d.c. value) }\end{array}$ & $\begin{array}{c}60 \\
{[\text { Fig. } 10(a)]}\end{array}$ & $\mathrm{F}_{\text {Is }}-0.5$ \\
\hline $\begin{array}{l}\text { Frequency of } \\
\text { Operation }\end{array}$ & $\begin{array}{c}10.7 \mathrm{GHz} \\
{\left[(3 / 4) \cdot\left(\mathrm{v}_{\mathrm{s}} / \mathrm{w}\right)\right]}\end{array}$ & $\begin{array}{c}10.5 \\
\text { (rable 2) }\end{array}$ & $F_{f} \sim 1.0$ \\
\hline $\begin{array}{l}\text { Maxinum. Fower } \\
\text { Density }\end{array}$ & $\begin{array}{c}24,000 \mathrm{w} / \mathrm{cm}^{2} \\
(\mathrm{Eq} . \mathrm{l})\end{array}$ & $\begin{array}{c}260 \\
\text { (Table 2) }\end{array}$ & $F_{p}-0.01$ \\
\hline $\begin{array}{l}\text { Induced Current } \\
\text { Tapering }\end{array}$ & $\begin{array}{l}\text { rectangular } \\
\text { (Fig. 7) }\end{array}$ & $\begin{array}{l}\text { tapered end } \\
\text { [Fig. } 10(\mathrm{~d})]\end{array}$ & $F_{i c}-0.2$ \\
\hline
\end{tabular}

Table 3. Power reduction factors 


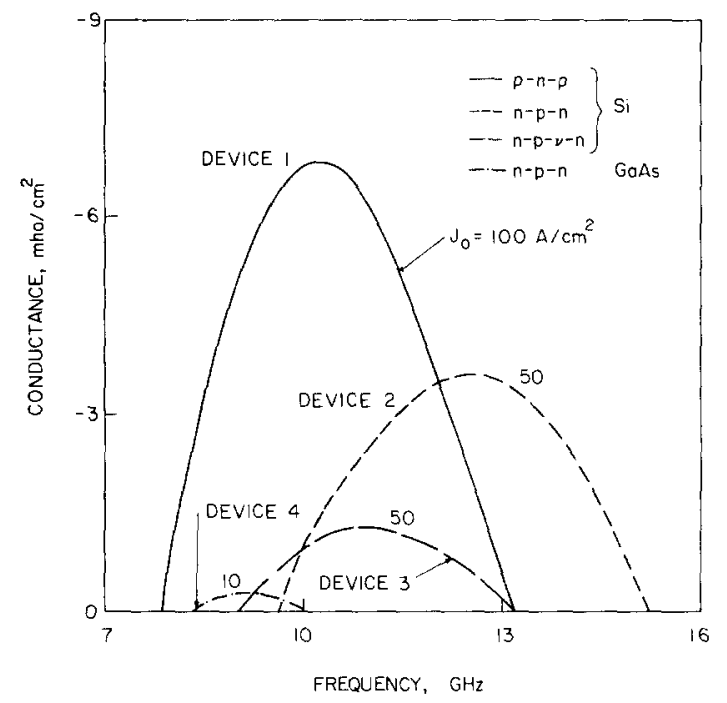

Fig. 14. Small-signal conductance at the optimum current densities of devices 1 through 4.

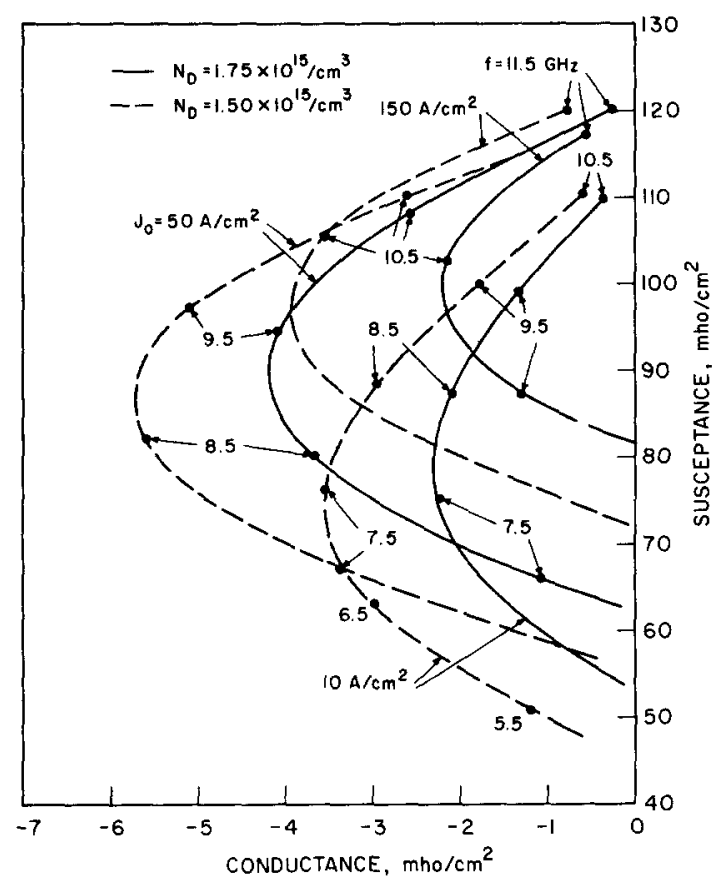

Fig. 15. Effect of doping density on admittance of a $p-n-p$ structure. $w=6.3 \mu \mathrm{m}$.

the carriers. In a transit-time device, for a given frequency, the width of the drift region is increased proportionately to the saturated velocity. The higher the value of the carrier saturated velocity the larger is the width of the device. Hence, the voltage and powerhandling capability of the device is increased accordingly.

The low-field effect is controlled by the low-field mobility of the carriers. Such influence can be seen from the numerical results of the Si $p-n-p$ and $n-p-n$ structures listed in Table 2 . The two structures are identical in all respects except in the mobility expressions where the low-field mobility for holes [15] is $333.3 \mathrm{~cm}^{2} / \mathrm{V} \cdot \mathrm{s}$ and for electrons, $1300 \mathrm{~cm}^{2} / \mathrm{V} \cdot \mathrm{s}$. The results show that the high-mobility value of electrons: (1) reduces the value of the optimum d.c. current density, (2) increases the RF voltage handling capability and (3) increases the operating frequency.

The high-mobility value of electrons not only increases operating frequency but also allows the injected carriers to be swept out of the injection region rapidly, thereby increasing the level of large-signal injection before the space-charge-limited injection reduces the output power. On the other hand the high-mobility value reduces both the value of the d.c. current density where the negative conductance is maximum and also the magnitude of the negative conductance itself. As shown in Fig. 14, the optimum d.c. current density is $50 \mathrm{~A} / \mathrm{cm}^{2}$ for the $n-p-n$ structure as compared to $100 \mathrm{~A} / \mathrm{cm}^{2}$ for the $p-n-p$ one, and the maximum value of negative conductance is $3.6 \mathrm{mho} / \mathrm{cm}^{2}$ for the $n-p-n$ structure as compared to $6.8 \mathrm{mho} / \mathrm{cm}^{2}$ for the $p-n-p$ one.

For the GaAs $n-p-n$ structure, the exceedingly high value of the low-field mobility of electrons drives down drastically the value of the optimum current density as well as the value of negative conductance. As shown in Fig. 14, the optimum current density is $10 \mathrm{~A} / \mathrm{cm}^{2}$ with the maximum negative conductance approximately equal to $0.26 \mathrm{mho} / \mathrm{cm}^{2}$ which is more than one order of magnitude less than that of the Si structures. As a result the GaAs $n$ $p-n$ structure is difficult to operate as a BARITT device.

\section{CONCLUSIONS}

The results of first-order small-signal and large-signal analyses of BARITT devices were compared with those obtained numerically by an accurate computer simulation. It was found that the small-signal analysis with an assumption of a constant saturated drift velocity tends to predict a significantly higher frequency of operation than that obtained numerically. This is especially true for the uniformly doped BARITT structure. Also the small-signal conductance and the optimum bias current density as well as its optimum frequency of operation are sensitive to the adopted model of current injection. This suggests a need for a judicious modification of the analysis in order for it to be accurate.

The first-order large-signal theory [1] predicted that the Si BARITT device is capable of generating $24,000 \mathrm{~W} / \mathrm{cm}^{2}$ of power at $10 \mathrm{GHz}$ with a maximum efficiency of $21 \%$. The results of numerical calculations indicated a maximum power capacity of approximately $300 \mathrm{~W} / \mathrm{cm}^{2}$ with a maximum efficiency of $3 \%$. Suggestions were made that there are four factors which can account for this large discrepancy. They are:

1. The space-charge-limited injection factor which severely constricts the magnitude of the induced current.

2. The induced current tapering factor which can be traced to the combined effects of spatial velocity modulation, carrier diffusion and premature carrier collection at various phases of the cycle.

3. The large-signal degradation factor which arises from large-signal field depression in the drift region.

4. The frequency reduction factor due to the slow carrier packet motion in the low field region and to a lesser 
extent due to the electric field depression in the drift region.

For an optimized $\mathrm{Si} n-p-\nu-n$ structure with a $7-\mu \mathrm{m}$ wide drift region, the space-charge-limited injection accounts for power reduction of a factor of ten; the induced current tapering, a factor of five; the large-signal degradation, a factor of two; and the frequency reduction, a factor of unity. Since the magnitude of the induced current is proportional to the d.c. bias current, the device efficiency reduction factor is equal to the product of the last three reduction factors, which is ten.

Finally, the effects of doping profile and material parameters on the properties of BARITT devices were investigated numerically. It was found that the Read ( $n-$ $p-\nu-n)$ structure with a narrow, heavily doped $p$-region increases the device large-signal handling capacity. However, due to the high doping density of the $p$-region the magnitude of the negative conductance is reduced. Consequently, the maximum power and efficiency of the Read structure is only comparable to a well-designed uniformly doped $(n-p-n)$ structure.

The effects of the low-field mobility upon the performance of BARITT devices are more complex. As has been shown numerically the high value of low-field mobility allows the injected carriers to move rapidly in the low-field region resulting in a higher operating frequency and also in a higher large-signal handling capability before the detrimental space-charge effect dominates. On the other hand, this high value is accompanied by a lowering of the optimum d.c. current density. Consequently, a device with a high low-field mobility normally is operated at a low current density with a large $R F$ voltage amplitude. Its negative conductance is small but it degrades slowly with the signal amplitude. The exceedingly high value of electron mobility in GaAs renders the GaAs $n-p-n$ structure difficult to operate as a BARITT device.
It is also worth noting that even though the BARITT device is inferior to other solid-state devices with regard to power output and efficiency, its low noise and low conversion loss make it an extremely useful device in self-mixed doppler radar applications [19].

\section{REFERENCES}

1. H. W. Rüegg, IEEE Trans. Electron Devices ED-15, 577 (1968).

2. C. P. Snapp and P. Weissglas, IEEE Trans. Electron Devices ED-19, 1109 (1972).

3. J. Helmcke, H. Herbst, M. Claassen and W. Harth, Electronics Letters 8, 158 (1972).

4. S. P. Kwok, Properties and Potential of BARITT Devices, Tech. Report No. 133. Electron Physics Laboratory, The University of Michigan, Ann Arbor (1974).

5. D. L. Scharfetter and H. K. Gummel, IEEE Trans. Electron Devices ED-16, 64 (1969).

6. H. A. Haus, H. Statz and R. A. Pucel, Electronics Letters 7, 667 (1971).

7. D. J. Coleman, Jr., J. Appl. Phys. 43, 1812 (1972).

8. K. P. Weller, RCA Rev. 32, 372 (1971).

9. J. L. Chu and S. M. Sze, Solid-St. Electron. 16, 85 (1973).

10. G. T. Wright, Solid-St. Electron. 16, 903 (1973).

11. A. Sjölund, Solid-St. Electron. 16, 559 (1973).

12. J. G. Oakes and C. A. Lee, Int. Electron Devices Meeting Tech. Digest, Washington, D.C., pp. 479-482 (1973).

13. W. T. Read, Jr., Bell Syst. Tech. J. 37, 401 (1958).

14. S. M. Sze, D. J. Coleman, Jr, and A. Loya, Solid-St. Electron. 14, 1209 (1971).

15. J. L. Chu, G. Persky and S. M. Sze, J. Appl. Phys. 43, 3510 (1972).

16. Z. Kopal, Numerical Analysis, pp. 145-150. Chapman and Hall, London (1955).

17. C. A. Lee, R. A. Logan, R. L. Batdorf, J. J. Kleimack and W. Wiegmann, Phys. Rev. 134, A761 (1964).

18. R. A. Logan and S. M. Sze, J. Phys. Soc. Japan 21, Suppl., 434 (1966).

19. S. P. Kwok, H. Nguyen-Ba and G. I. Haddad, Presented at the 1974 Int. Solid-State Circuits Conf., Philadelphia, PA (1974). 